\title{
Pointing and Anti-pointing in Müller-Lyer figures: Why illusion effects need to be scaled.
}

\author{
Constanze Hesse ${ }^{1}$, Volker H. Franz ${ }^{2}$ \& Thomas Schenk ${ }^{3}$
}

$03 / 08 / 2015$

keywords: perception, action, illusion, delay, anti-pointing

RUNNING TITLE: "Illusion effects in pointing and anti-pointing"

Correspondence should be addressed to:

Dr. Constanze Hesse

School of Psychology

University of Aberdeen

King's Campus

AB24 3FX

United Kingdom

e-mail: c.hesse@abdn.ac.uk

phone: ++44 (0)1224 273215

\footnotetext{
${ }^{1}$ School of Psychology, University of Aberdeen, UK

${ }^{2}$ Department of Psychology, University of Hamburg, Germany

${ }^{3}$ Department of Neuropsychology, Ludwig-Maximilians-University, Munich, Germany
} 


\section{$\underline{\text { Abstract }}$}

It was suggested that goal-directed actions performed under full-vision are immune to certain visual illusions, while movements relying on perception-based visual information are deceived by them (Milner \& Goodale, 1995). Consequently, pointing movements should be deceived by visual illusions when a delay is introduced (memory demands) or when anti-pointing (spatial imagery) is required. In two experiments, participants performed either pro-pointing or anti-pointing movements to different versions of the Müller-Lyer illusion in two vision conditions (open-loop vs. delay). Apart from open-loop pro-pointing, all conditions should rely on perceptual processing and should therefore yield similarly illusion effects. While we observed illusion effects in all conditions, their magnitude varied in unexpected ways. Most surprisingly, introducing a delay seemed to reduce illusion effects in anti-pointing. We show that this decrease can be explained by the fact that pointing after delay is less responsive to physical size changes. After correcting for this, illusion effects in anti-pointing were similar in both vision conditions but still twice as large as in the delayed pro-pointing task. Our findings highlight the necessity of employing a correction procedure when comparing illusion effects across tasks and do not conform well to the predictions derived from the perception-action model.

Keywords: perception, action, illusion, delay, anti-pointing 


\section{$\underline{\text { Introduction }}$}

Since the influential study by Aglioti, DeSouza, and Goodale (1995) in which they concluded that visual illusions have a negligible effect on our actions in spite of strongly affecting our perception of a stimulus, the question of whether or not these findings provide behavioural evidence in favour of the perception-action model has been discussed controversially (for review see, Franz \& Gegenfurtner, 2008; Goodale \& Westwood, 2004; Mclntosh \& Schenk, 2009; Milner \& Goodale, 2006; Schenk, Franz, \& Bruno, 2011). The underlying rationale for Aglioti's study was that, according to the perception-action model, visual information involved in the identification and recognition of objects and visual information for the guidance of actions are processed differently and in divergent cortical pathways (Milner \& Goodale, 1995, 2006). Specifically, it is assumed that visual information used for perception is processed in the ventral pathway. According to the model, ventral stream information is consciously accessible, represented viewpoint independently (allocentric frame of reference) and stored over longer periods in time. In contrast, visual information for action guidance is assumed to be processed in the dorsal pathway that represents information metrically accurate, on a moment to moment basis and relative to the observer (egocentric frame of reference). Consequently, it is predicted that perception but not action is deceived by visual illusions. While initial studies seemed to support this prediction (Aglioti et al., 1995; Haffenden \& Goodale, 1998), the validity of the findings was soon questioned by numerous researchers (Franz, Gegenfurtner, Bülthoff, \& Fahle, 2000; Pavani, Boscagli, Benvenuti, Rabuffetti, \& Farnè, 1999; Smeets, Brenner, de Grave, \& Cuijpers, 2002). In short, critics pointed out that, in most cases, the observation that illusion effects are smaller in visuomotor tasks than in perceptual tasks can be attributed to methodological problems and/or statistical artefacts (see, Dassonville \& Bala, 2004; Franz, 2001; Franz \& Gegenfurtner, 2008; Mon-Williams \& Bull, 2000; Pavani et al., 1999; Post \& Welch, 1996; Smeets \& Brenner, 2006; Smeets et al., 2002). 
However, in addition to the debated issue of whether illusion effects are similar in perception and action, the perception-action models also makes clear predictions about situations in which actions can be expected to be susceptible to visual illusions. In other words, the perception-action model suggests that in certain conditions the metrically accurate visual representation of the dorsal stream is unavailable for action guidance. In these cases visuomotor processing has to rely on ventral stream processing. A typical situation in which this would be the case is when no visual information is available during action programming (Goodale, Kroliczak, \& Westwood, 2005; Goodale, Westwood, \& Milner, 2004; Westwood \& Goodale, 2003). As the dorsal stream is assumed to work in real-time, visual representations for the control of actions are not stored. Hence, if vision is suppressed before a movement is initiated the motor system has to rely on the stored ventral representation resulting in a susceptibility of motor actions to visual illusions. Remarkably, all studies that measured grasping (or pointing movements) in conditions with varying pre-movement delays consistently reported larger illusion effects for movements performed after delay than for movements performed without a delay (e.g., Franz, Hesse, \& Kollath, 2009; Gentilucci, Chieffi, Daprati, Saetti, \& Toni, 1996; Hu \& Goodale, 2000; Westwood, Heath, \& Roy, 2000; Westwood, McEachern, \& Roy, 2001). However, even though these findings are at first glance in agreement with the predictions of the perception-action model, it was proposed by Franz et al. (2009) that the size of the illusion effect on grasping may be better explained by the availability of visual feedback during grasping than by a shift from dorsal to ventral stream control after delay (for similar argument see, Mon-Williams \& Bull, 2000; Post \& Welch, 1996). In other words, while findings are relatively consistent the underlying causes are discussed controversially.

Another type of movements that is assumed to be mediated by the visuo-perceptual networks of the ventral stream are anti-pointing movements (Bruno \& Bernardis, 2003; Heath, Maraj, Gradkowski, \& Binsted, 2009; Maraj \& Heath, 2010). Anti-pointing requires the participant to perceive a target positioned relative to a landmark (or their hand) and then perform a movement in the mirrorsymmetrical direction. Similar to anti-saccades, anti-pointing movements show considerably longer 
reaction times than normal target-directed movements (pro-pointing) suggesting that they require enhanced top-down processing. Recently, Heath et al. (2009) observed that anti-pointing movements show a similar endpoint bias as perceptual judgments. Based on this finding, they suggested that anti-pointing movements are cognitively driven and thus controlled by perceptual rather than by visuomotor representations. In a follow-up study, the authors also showed that antipointing is likely to be performed in offline-mode of control (Maraj \& Heath, 2010) further supporting the notion that anti-pointing movements are mediated by the perceptual networks.

Within the framework of the perception-action model, illusion effects are seen as indicators of the involvement of the ventral stream. The presence of an illusion effect in a given task suggests that this task is served by visual information from the ventral stream; its absence suggests that the task relies on information from the dorsal stream. Both delayed movements and anti-pointing movements are assumed to be served by ventral stream information and thus both tasks should be affected by illusions in a similar way. Given this logic, we would also expect that the effects of introducing a delay and of performing anti-pointing movements instead of pro-pointing movements are non-additive. As both tasks are assumed to rely on the same illusion-distorted ventral-stream representation, combining the two tasks should not increase the distortion and should thus leave the size of the illusion effect unaffected. The current study was designed to test these predictions. This is particularly important, as it will allow further insights into the controversial issue of whether or not delayed movements are mediated by the visuo-perceptual ventral stream (e.g., Fiehler et al., 2011; Heath, Neely, Krigolson, \& Binsted, 2010; Hesse \& Franz, 2009; Hesse \& Schenk, 2014; Himmelbach \& Karnath, 2005).

We asked participants to perform pro- and anti-pointing movements to versions of the Müller-Lyer illusion in two different viewing conditions (note that this size illusion has been frequently used to test for perception-action dissociations; for review see Bruno, Bernardis, and Gentilucci (2008) on pointing movements, and Bruno and Franz (2009) on grasping movements). In the open-loop 
viewing condition, vision was available during the movement programming phase thus ensuring that the real-time visuomotor networks of the dorsal stream were involved in movement programming. In the delay condition, a two second delay was introduced between viewing the target and the initiation of the motor response. Hence, movement programming had to supposedly rely on the stored representation of the ventral stream. The paradigm allowed us to test the prediction (based on the assumptions of the perception-action model) that memory guided movements and cognitively driven motor responses (anti-pointing) are based on the same visual representations and are thus similarly susceptible to visual illusions.

\section{Experiment 1}

\section{Methods}

Participants: Sixteen volunteers participated in the experiment ( 6 male, mean age: 26 years, age range: $20-40$ years). All participants were right-handed by self-report, had normal or corrected-tonormal vision and were naïve with respect to the purpose of the study. The experiment was approved by the local ethics committee and written consent was obtained from each participant before the beginning of the experiment.

Setup: Participants were seated comfortably on a height-adjustable chair at a table within a dimly lit room. In front of them, an IPS (in-plane switching) computer monitor (Dell P1914S, $1280 \times 1024$ pixel, $30 \mathrm{~cm} \times 37.5 \mathrm{~cm}, 60 \mathrm{~Hz}$ ) was screwed flatly to the table surface in vertical orientation (portrait mode) at a viewing distance of about $50 \mathrm{~cm}$. A thin plexiglas panel $(30 \mathrm{~cm} \times 37.5 \mathrm{~cm} \times 0.3 \mathrm{~cm})$ was placed on the surface of the monitor to protect the screen.

Pointing movements were recorded with an Optotrak 3020 system (Northern Digital Incorporation, Waterloo, Ontario, Canada) at a sampling rate of $200 \mathrm{~Hz}$. One infrared light-emitting diode was attached to the nail of the right index finger. Prior to the experiment, the system was calibrated such that the Cartesian coordinate system was aligned with the plane of the monitor. To control the 
visibility of the stimuli presented on the computer screen, participants wore liquid-crystal shutter glasses (PLATO Translucent Technologies, Toronto, Ontario; Milgram (1987)), which rapidly suppress vision by changing from a transparent to an opaque state. The experiment was programmed in MATLAB using the Psychophysics Toolbox (Brainard, 1997; Kleiner, 2010) and the custom-built Optotrak Toolbox (Franz, 2004b).

Stimuli: The stimulus was a vertical version of the Müller-Lyer Illusion (ML-Illusion) with a shaft length of either $7 \mathrm{~cm}$ or $10 \mathrm{~cm}$. The length of the fins was determined as $25 \%$ of the shaft length. The angle of each fin with respect to the shaft was $30^{\circ}$ for inward fins and $150^{\circ}$ for outward fins. The thickness of all lines was $1 \mathrm{~mm}$. All stimuli were presented in white on a dark grey background. The ML-Illusion was presented so that one end of the figure was aligned with the centre of the screen. The opposite end of the ML-figure could either be directed downward (toward the participant) or upward away from the participant from the middle of the screen (see Figure 1).

Procedure: Before the beginning of each trial shutter glasses opened and participants were instructed to place their right index finger on the plexiglas at the starting position displayed in the centre of the screen (black cross, $0.6 \mathrm{~cm} \times 0.6 \mathrm{~cm})$. An armrest $(29 \mathrm{~cm} \times 9 \mathrm{~cm} \times 7 \mathrm{~cm}$ ) was positioned on the right side of the monitor so participants could rest their hand comfortably. Once participants had placed their finger on the start position the experimenter closed the shutter glasses by pressing a key. Then the experimenter initiated the trial with another key press. Subsequently, the ML-figure was presented on the screen and the shutter glasses changed to transparent state for a preview period of $500 \mathrm{~ms}$. Participants were instructed to view the illusion during this preview period but to wait with their movements until a go-signal $(1000 \mathrm{~Hz}, 100 \mathrm{~ms})$ was presented. As one end of the MLfigure was always aligned with the centre of the screen, the start position corresponded to one end of the illusion. There were four different blocks of trials that varied the nature of the required pointing response (pro-pointing vs. anti-pointing) as well as the amount of available visual information and memory demands (open-loop vs. delay). 
In the pro-pointing task, participants were instructed to point as accurately as possible to the perceived opposite end of the shaft of the ML-figure requiring either a movement away or toward the body from the start position (Figure 1A). In the anti-pointing task, participants were asked to point to a virtual point that was relative to their start position in direction and distance opposite to the endpoint of the ML-figure (Figure 1B). Participants were instructed to point as accurately as possible requiring either a movement away or toward the body. In all conditions participants were allowed $2 \mathrm{~s}$ to execute the pointing movement. Both pointing tasks were combined with either an open-loop or a delay viewing condition.

In the "open-loop" condition (OL), the go-signal was given directly after the preview period and shutter glasses turned opaque when the index finger was moved away from the start position (i.e., $15 \mathrm{~mm}$ in y-direction). That is, in this condition the closing of the shutter glasses was triggered by the movement of the index finger and participants pointed without seeing either the stimulus or their hands. In contrast, in the "delay-condition" (DL) the shutter glasses closed directly after the preview period and there was a 2 second delay until the go-signal was presented. The shutter glasses remained opaque throughout the movement meaning that participants could not see their hand during the movement and had to remember the stimulus throughout the delay period and the duration of the movement (Figure 1).

All four conditions (pro-pointing open-loop, pro-pointing delay, anti-pointing open-loop, and antipointing delay) were blocked and counterbalanced across participants. Within each block, the presented versions of the ML-figure (fins-in vs. fins-out), the different shaft-lengths $(7 \mathrm{~cm}$ vs. $10 \mathrm{~cm})$ and the different display directions (up vs. down) were randomised. Each stimulus was presented six times resulting in 48 trials per block. Each block was preceded by four practice trials to familiarise participants with the task. After the completion of each block participants were allowed a short five minute break. The experiment took about one hour to complete. 
Data analysis: Movement data was filtered offline using a second-order Butterworth Filter with a low-pass cut-off frequency of $15 \mathrm{~Hz}$. For each trial, we determined the following parameters: reaction time (RT), movement time (MT) and the size of the illusion effect (IE). Movement onset was determined by a velocity threshold. The first frame in which the marker on the index finger exceeded a velocity of $0.05 \mathrm{~m} / \mathrm{s}$ was taken as movement onset. RT was defined as the time between the go-signal and movement onset. Similarly, movement offset was defined as the moment the velocity of the index marker dropped below a threshold of $0.025 \mathrm{~m} / \mathrm{s}$. MT was defined as the time between movement onset and movement offset.

To calculate the illusion effect, we determined the movement amplitude as the distance covered by the index finger in y-direction from the start position to the end of the movement. Then, we calculated the difference between the mean movement amplitude for outward and inward fin configurations separately for both shaft lengths and pointing directions for each participant. Finally, illusion effects were averaged across the two movement directions and the two different shaft lengths.

If not stated differently, data was analysed using repeated-measures ANOVAs with the withinsubject factors movement condition (pro-pointing vs. anti-pointing) and vision condition (OL vs. $2 \mathrm{sD})$. Values are presented as mean \pm 1 standard error of the mean. A significance level of $\alpha=.05$ was used for all statistical tests. If multiple tests were applied, p-values were Bonferroni corrected.

Correction of illusion effects (Scaling for slope of response function):

In order to obtain unbiased measures of the illusion effects in all conditions, we computed scaled (or corrected) illusion effects. Several researchers have argued, for almost 15 years now, that illusion effects need to be corrected by scaling them with the slope of the linear regression function that relates the dependent variable (e.g., hand opening or pointing distance) to physical variations of the target sizes used in the experiment (Bruno \& Franz, 2009; Dewar \& Carey, 2006; Franz, 2003a, 
2003b; Franz, Scharnowski, \& Gegenfurtner, 2005; Glover \& Dixon, 2002; Schenk et al., 2011). The rationale for this correction procedure is simple: If you want to measure how an observed behaviour (dependent variable) changes in response to an illusory change in size, you first have to know how much this behaviour (dependent variable) responds to physical changes in target size. For example, it is well know that in grasping the maximum hand opening increases by about $0.8 \mathrm{~mm}$ when the target size is increased by $1 \mathrm{~mm}$ (Smeets \& Brenner, 1999). In contrast, in another task in which participants are asked to indicate how large a target is by adjusting the opening between their index finger and thumb (manual estimation) an increase in target size by $1 \mathrm{~mm}$ leads to a $1.6 \mathrm{~mm}$ increase in the measured hand opening (Franz, 2003a; Haffenden, Schiff, \& Goodale, 2001). Hence, if we were to introduce an illusory size change of $2 \mathrm{~mm}$, we would expect to find an illusion effect of 1.6 $\mathrm{mm}$ in the grasping response but of $3.2 \mathrm{~mm}$ in the manual estimation response. In this case, the difference between the two measures does not reflect that grasping is less deceived by the illusion but that grasping is in general less responsive to changes in target size than manual estimation. An easy way to account for these differences in response functions between different tasks is to measure the illusion effect in the different conditions and then divide it by the slope of the response function measured for physical (non-illusory) size changes in the same condition.

$$
C I E=\frac{i}{S}
$$

with, CIE: corrected illusion effect, $i$ : mean illusion effect, $s$ : mean slope.

Note, that this procedure is equivalent to the calibration of a scientific instrument (e.g., JCGM-200, 2012; Miller, 1986). To see why, consider we want to craft a milometer for a bicycle that simply counts the number of turns of one of the wheels. To calibrate this milometer, we would begin by cycling known distances and determine the relationship between the known distances travelled and the indications measured by the milometer. Because the milometer behaves roughly linear, the first step of the calibration is to determine a regression line: 


$$
\text { Indication }=a+s * \text { Dist }_{\text {travelled }}
$$

With, a: intercept, s: slope, Dist: Distance.

In the second step of the calibration ${ }^{1}$, we would travel an unknown distance and use the indication of the milometer to measure this distance. For this, we need to rearrange the regression function:

$$
\text { Dist }_{\text {unkown }}=\frac{(\text { indication }-a)}{s}
$$

Quite often this formula can be simplified further as we are only interested in the difference between two unknown distances. In this case, the intercepts would cancel out (i.e., intercept intercept $=0$ ) and the formula reduces to:

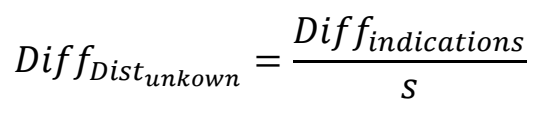

This formula is the exact analogue of the one we use to compute our corrected illusion effects: We first determine the response of a dependent variable to known variations of physical size and calculate the corresponding regression line (importantly the relevant dependent variables are known to behave linearly). Second, we present an unknown change in size (which is caused by the illusion) and use the regression line to measure the size of the illusion effect.

Applying this reasoning to our current study, we first determined for each condition and participant the slopes of the response functions that relate pointing distance to physical stimulus length. Based on previous studies, we know that pointing distance is linearly related to target length (e.g., Prablanc, Echallier, Komilis, \& Jeannerod, 1979; Soechting \& Flanders, 1989). Using these slopes, we then scaled the measured illusion effects to obtain a corrected measure for the size of the illusion.

Although calculating the corrected illusion effects is very simple, calculating the corresponding standard errors and confidence intervals is not trivial, as both the variability of the measured illusion

\footnotetext{
${ }^{1}$ Although often the first step alone is perceived as being calibration, the definition of calibration comprises both steps (JCGM, 2012)
} 
effect as well as the variability of the measured slopes (which are in the denominator of the above formula) need to be taken into account. Problems arise if the slopes are very close to zero as the confidence limits for the corrected illusion effects will become infinite and thus the corrected illusion effects will be arbitrarily large (or small). This is a well-known problem from the literature on calibration and was first described by Fieller (1940). However, calculations become much simpler if, as in our case, the measured slopes are significantly different from zero. In this case, standard errors and confidence intervals can be calculated using an approximation based on the Taylor expansion (for details see Buonaccorsi (2001) and Franz (2007)). This results in:

$$
S E_{C I E}=\left|\frac{i}{s}\right| \sqrt{\frac{1}{N}\left(\frac{\sigma_{s}^{2}}{s^{2}}+\frac{\sigma_{i}^{2}}{i^{2}}-2 \frac{\sigma_{i s}}{i s}\right)}
$$

with, $S E_{C I E}$ : standard error of corrected illusion effect, $i$ : mean illusion effect, $s$ : mean slope, $\sigma_{s}$ : standard deviation of slope, $\sigma_{i}$ : standard deviation of illusion effect, $\sigma_{i s}$ : covariance of illusion effect and slope, $N$ : number of participants.

It can be practical to rearrange the above formulas such that we obtain for each participant an individual Taylor-approximated value (e.g., if a statistical program requests a data entry per participant; this rearrangement does, however, not change the result of the calculations):

$$
C I E_{k}=\frac{i}{S}\left(1-\frac{s_{k}}{S}+\frac{i_{k}}{i}\right)
$$

with, $\mathrm{ClE}_{\mathrm{k}}$ : individual taylor-approximated illusion effect for participant $\mathrm{k}, \mathrm{i}=$ mean illusion effect, $\mathrm{s}=$ mean slope, $\mathrm{i}_{\mathrm{k}}=$ individual illusion effect of participant $\mathrm{k}$, and $\mathrm{s}_{\mathrm{k}}=$ individual slope of participant $\mathrm{k}$.

In a nutshell, illusion effects cannot validly be compared across conditions when the corresponding slopes (that relate target size to pointing distance) vary. Hence, corrected (or scaled) illusion effects are calculated in order to account for the differences in slopes and obtain a valid measure for how the illusion affected pointing movements in the different conditions. This is done by dividing the observed illusion effect by the slope. Throughout this article we will report both uncorrected and corrected illusion effects. 


\section{Results and Discussion}

\section{Illusion Effects:}

Figure $2 \mathrm{~A}$ shows the movement amplitude measured for the two tasks, vision conditions, MüllerLyer configurations and shaft lengths. As expected, participants adjusted their movement amplitude for the different shaft lengths with slopes that were significantly larger than 0 (all $p<.001$ ). The average slopes were: $0.99 \pm 0.03$ for pro-pointing open-loop, $0.94 \pm 0.05$ for anti-pointing open-loop, $0.82 \pm 0.05$ for pro-pointing delay and $0.76 \pm 0.05$ for anti-pointing delay, pooled across the two different Müller-Lyer configurations (fins-in vs. fins-out). The 2 (task: pro-pointing vs. anti-pointing) $x$ 2 (vision condition: open-loop vs. delay) repeated measures ANOVA on the slopes revealed that the slopes were significantly smaller in the delay condition than in the open-loop vision condition, $F(1,15)=42.21, p<.001, \eta_{p}^{2}=.74$. There was no main effect of task $(p=.15)$ and no interaction effect $(p=.95)$.

Illusion effects are shown separately for both shaft lengths $(7 \mathrm{~cm}$ and $10 \mathrm{~cm})$ in Table 1 . For further analysis, we averaged the uncorrected illusion effects across both shaft lengths (see Figure 2B). Onesample t-tests confirmed that illusion effects in all conditions were significantly different from zero (all $p<.001$ ). The 2 (task: pro-pointing vs. anti-pointing) $\times 2$ (vision condition: open-loop vs. delay) repeated measures ANOVA on the uncorrected data showed a highly significant main effect of task, $F(1,15)=240.7, p<.001, \eta_{p}^{2}=.94$, indicating that illusion effects were much stronger in the antipointing condition $(31.7 \mathrm{~mm} \pm 1.5 \mathrm{~mm}$ ) than in the pro-pointing condition $(12.5 \mathrm{~mm} \pm 1.3 \mathrm{~mm})$. There was also a highly significant interaction effect, $F(1,15)=59.3, p<.001, \eta_{p}^{2}=.80$. Post-hoc tests suggested that illusion effects increased after delay in the pro-pointing condition, $t(15)=5.41$, $p<.001, d=1.35$, but significantly decreased after delay in the anti-pointing condition, $t(15)=4.30$, $p=.001, d=1.06$. There was no main effect of delay $(p=.95)$. 
However, before attempting any theoretical interpretations of this rather unpredicted finding (i.e., in particular the decreased illusion effects after delay in anti-pointing), we considered it crucial to look at the corrected illusion effects. As discussed in the methods section of this paper as well as in previous studies (e.g., Franz et al., 2001; Franz et al., 2008), the effect of a size illusion will depend on the response of the perceptual and/or motor system to the physical variations in the size of the stimulus (specifically the shaft length in this case). As shown above, there was a significant effect of delay on the slopes. This means that after delay participants adjusted their pointing movements less in response to physical changes in size of the stimulus. Specifically, our data shows that a $10 \mathrm{~mm}$ increase in the physical shaft length resulted in a $9.4 \mathrm{~mm}$ increase of the pointing amplitude in the anti-pointing open-loop condition but only a $7.6 \mathrm{~mm}$ increase in the anti-pointing delay condition. Hence, if the illusion induces an average illusory size change of about $30 \mathrm{~mm}$ between the fins-in and fins-out configuration in the anti-pointing task then participants would be expected to show an illusion effect of about $28.2 \mathrm{~mm}(30 \times 0.94 \mathrm{~mm})$ in the open-loop condition but only of about 22.8 $\mathrm{mm}(30 \times 0.76 \mathrm{~mm})$ in the delay condition. This may explain the unexpected decrease in the illusion effect after delay in anti-pointing. Note, that it would also mean that in the pro-pointing task the illusion effects after delay is even more pronounced than suggested by the uncorrected effects (see methods section for more details).

Corrected illusion effects are depicted in Figure 2C. Interestingly, after the correction was applied illusion effects were virtually identical for the open-loop and delayed anti-pointing conditions. Paired-samples t-tests confirmed that the scaled illusion effect was significantly increased after delay in the pro-pointing condition, $t(15)=7.77, p<.001, d=1.94$ while there was no effect of delay on the scaled illusion effect in the anti-pointing task, $t(15)=0.09, p=.93, d=0.02$. Thus, if illusion effects are corrected such that participants' responsiveness to physical changes in shaft size is taken into account, illusion effects in anti-pointing are, as predicted, unaffected by the introduction of a delay, but surprisingly remained about twice as large as in delayed pro-pointing. 


\section{$\underline{R T \text { and } M T:}$}

Finally, we also looked at the temporal aspects of the pointing movement (RT and MT). The 2 (task: pro-pointing vs. anti-pointing) $\times 2$ (vision condition: open-loop vs. delay) repeated measures ANOVA on the RT-data showed a significant main effect of task, $F(1,15)=7.12, p=.018, \eta_{p}^{2}=.32$. Post-hoc tests indicated that movements were initiated faster in the pro-pointing conditions ( $349 \mathrm{~ms} \pm 19 \mathrm{~ms}$ ) than in the anti-pointing conditions ( $375 \mathrm{~ms} \pm 21 \mathrm{~ms}$ ). There was no main effect of vision condition $(p=.31)$, but a significant interaction effect, $F(1,15)=5.20, p=.038, \eta_{p}^{2}=.26$. While participants were quicker to initiate their movements in the pro-pointing open-loop condition ( $322 \mathrm{~ms} \pm 22 \mathrm{~ms}$ ) than in the pro-pointing delay condition ( $376 \mathrm{~ms} \pm 24 \mathrm{~ms}$ ), they were similarly fast in both vision conditions of the anti-pointing task (open-loop: $383 \mathrm{~ms} \pm 25 \mathrm{~ms}$ vs. delay: $367 \mathrm{~ms} \pm 21 \mathrm{~ms}$ ).

The same analysis on the MT-data revealed again a significant main effect of task, $F(1,15)=13.84$, $p=.002, \eta_{p}^{2}=.48$, as well as a significant main effect of vision condition, $F(1,15)=7.93, p=.013, \eta_{p}^{2}=.35$ There was no interaction effect $(p=.092)$. Post-hoc tests confirmed that movements were on average performed more slowly in the anti-pointing task (481 ms $\pm 35 \mathrm{~ms}$ ) than in the pro-pointing task (427 $\mathrm{ms} \pm 31 \mathrm{~ms}$ ) and more slowly in the delay condition (477 ms $\pm 36 \mathrm{~ms}$ ) than in the open-loop vision condition (430 ms $\pm 31 \mathrm{~ms})$.

The finding that both RTs and MTs were prolonged when anti-pointing was required is in line with previous research and supports the argument that anti-pointing requires higher-level cortical resources (Heath et al., 2009; Maraj \& Heath, 2010).

\section{Experiment 2}

The most surprising finding of Experiment 1 was that the illusion effects observed in both vision conditions of the anti-pointing task were almost twice the size of the illusion effects observed in the pro-pointing delay condition (Fig 2C). According to the predictions of the perception-action model both types of movements, anti-pointing and delayed pro-pointing, should be mediated by the 
perceptual representations of the ventral stream and should hence show similar susceptibility to visual illusions. Therefore, it seems that an explanation in terms of the perception-action model is problematic and consequently we need to look for an alternative account for our data.

In our opinion, it is likely that the observed effects and specifically the doubling of the illusion effect in anti-pointing can be best explained in terms of one of the existing theories on how the MüllerLyer illusion works. Specifically, it has been proposed that the Müller-Lyer illusion introduces two distortion effects: Firstly, a small misperception of the position of the intersections and secondly, a much larger misperception of the shaft extent (e.g., Gillam \& Chambers, 1985; Mack, Heuer, Villardi, \& Chambers, 1985; Smeets et al., 2002). In line with this suggestion, Predebon (2005) could show that pointing movements are more strongly affected by the Müller-Lyer illusion when pointing is based on extent information than when it is based on position information. In our study, the relevance of information about position and extent depended strongly on the performed task (propointing vs. anti-pointing), thereby influencing the size of the measured illusion effect (see also, Smeets et al., 2002 p. 141). In the pro-pointing conditions, participants can use information about position to determine the target location. In other words, in this task participants need to determine the end-point of the Müller-Lyer configuration that is opposite to the start-position of the hand. In contrast, in the anti-pointing task participants need to base their pointing response exclusively on an estimate of spatial extent as no direct information about target position is available. This estimate of spatial extent is strongly affected by the illusion and anti-pointing will hence show larger illusion effects. Finally, in the delayed pro-pointing condition the precision of the position estimate will decrease over time and hence participants may base their movements on a weighted combination of both position and extent information resulting in intermediate illusion effects.

A possible way to test this hypothesis would be to remove the fin-configuration presented at the start-position (that coincides with one end of the Müller-Lyer figure). Previous studies have reported that (perceptual) illusion effects on shaft extent tend to be halved in size when one of the fins- 
configurations is removed and the shaft length is kept constant (e.g., Predebon, 2000; Redding \& Hawley, 1993). Consequently, we would expect that the illusion effect in the anti-pointing task (that is based on the estimate of spatial extent) is halved in size when only the fin-configuration opposite to the start position is presented. In contrast, the position estimate should primarily depend on the fin configuration presented opposite to the start position. Thus, if the illusion effect in the open-loop pro-pointing task is primarily determined by a misperception of the fin position, the size of the illusion effect should be unaffected by whether or not fins are also present at the start position of the hand (for a different view see, Welch, Post, Lum, \& Prinzmetal, 2004). We tested these ideas by letting participants perform pointing movements in the same vision (open-loop and delay) conditions and movement conditions (pro-pointing and anti-pointing) as in Experiment 1 but this time only presented a fin configuration on the end of the Müller-Lyer figure to which participants had to point.

\section{Methods}

Participants: Twelve volunteers participated in the experiment ( 2 male, mean age: 28 years, age range: $19-40$ years) with eight of them having also participated in Experiment 1. All participants had normal or corrected-to-normal vision and were naïve with respect to the purpose of the study. One participant was left-handed and all participants performed the experiment with their dominant hand. The experiment was approved by the local ethics committee and written consent was obtained from each participant before the beginning of the experiment.

Setup, Stimuli, Procedure and Data analysis: The setup, experimental procedures and data analyses were identical to Experiment 1 . The target stimuli were changed such that there was only a fins-out or fins-in configuration visible at the far end of the line while no fins were presented at the start position of the finger. Like in Experiment 1, the length of the shaft was either $7 \mathrm{~cm}$ or $10 \mathrm{~cm}$.

\section{Results and Discussion}


Illusion Effects: Figure $3 \mathrm{~A}$ shows the average movement amplitude for the two tasks, vision conditions, Müller-Lyer configurations and shaft lengths. Similar as in Experiment 1, we tested how well participants adjusted their pointing movements to the two different shaft lengths by calculating the slope of the linear regression function that relates pointing distance to stimulus length. The 2 (task: pro-pointing vs. anti-pointing) $\times 2$ (delay: open-loop vs. delay) repeated measures ANOVA on the slopes revealed again a significant main effect of delay, $F(1,11)=30.29, p<.001, \eta_{p}^{2}=.73$. There was no main effect of task $(p=.21)$ and no interaction effect $(p=.16)$. The average slopes across all participants were $1.00 \pm 0.03$ for the pro-pointing open-loop task, $0.78 \pm 0.04$ for the pro-pointing delay task, $0.92 \pm 0.06$ for the anti-pointing open-loop task and $0.75 \pm 0.05$ for the anti-pointing delay task. All slopes were significantly different from zero (all $p<.001$ ).

The uncorrected illusion effects averaged across both shaft lengths are shown in Figure 3B (and separately for both shaft lengths in Table 1). In all conditions, illusion effects were significantly different from zero (all $p<.001$ ). Similar as in Experiment 1, the 2 (task: pro-pointing vs. anti-pointing) x 2 (delay: open-loop vs. delay) repeated measures ANOVA confirmed a significant effect of task on the size of the illusion effect, $F(1,11)=30.69, p<.001, \eta_{p}^{2}=.74$. Post-hoc tests confirmed that the illusion effect was larger in the anti-pointing task $14.0 \mathrm{~mm} \pm 0.9 \mathrm{~mm}$ than in the pro-pointing task 7.9 $\mathrm{mm} \pm 0.96 \mathrm{~mm}$. There was no main effect of delay $(p=.33)$ but again a significant interaction effect between task and delay, $F(1,11)=12.58, p=.005, \eta_{p}^{2}=.53$. Post-hoc tests indicated that there was no effect of delay on the size of the illusion effect in pro-pointing $(p=.32)$ but that uncorrected illusion effects again decreased with delay in anti-pointing, $t(11)=3.38, p=.006, d=0.97$.

As the responsiveness of the pointing movements to variations in shaft length considerably decreased after delay in both tasks (as indicated by the smaller slopes), it is again essential to calculate corrected illusion effects before interpreting the data. The corrected illusion effects are shown in Figure 3C. Notably, we could replicate our findings from Experiment 1 that illusion effects in anti-pointing are of similar size in the open-loop and in the delay conditions when the reduced 
slopes in the delay condition are taken into account, $t(11)=0.21, p=.84, d=0.06$. Furthermore, by correcting the data for the pro-pointing task, the expected effect of delay on the size of the illusion effect became apparent again, $t(11)=2.50, p=.03, d=0.72$.

There are two important implications of these findings. Firstly, by correcting the data, it becomes apparent that the findings, based on the uncorrected data (i.e. no effect of delay in pro-pointing and a beneficial effect of delay in anti-pointing), are caused by an overall reduction of the responseslopes to stimulus length after delay. Secondly, the pattern of results is consistent with our explanation based on a large effect of the Müller-Lyer illusion on extent and a small effect on position: The size of the illusion effect was a) approximately halved in the anti-pointing conditions (as anti-pointing is based on spatial extent and we halved the extent-illusion by removing one of the fins), b) unaffected in size in the open-loop pro-pointing condition (as open-loop pro-pointing is based on target position which remained unchanged by the removal of one fin), and c) reduced but not halved in the delayed pro-pointing condition (as for this condition the illusion effect is assumed to be a compromise between the reduced extent illusion and the unchanged position illusion). In short, these findings support our hypothesis that anti-pointing relies on an estimate of spatial extent while open-loop pro-pointing primarily relies on information about position. The observed intermediate illusion effects in the delayed pro-pointing task suggest that a weighted combination of both spatial extent and position information is used to determine the target location (please see the General Discussion section for more detail).

\section{$\underline{R T \text { and } M T}$}

The 2 (task: pro-pointing vs. anti-pointing) $\times 2$ (vision condition: open-loop vs. delay) repeated measures ANOVA on the RT-data showed a significant main effect of task, $F(1,11)=24.35, p<.001$, $\eta_{p}^{2}=.69$. As in Experiment 1, participants were slower in initiating their movements in the antipointing condition ( $348 \mathrm{~ms} \pm 24 \mathrm{~ms}$ ) than in the pro-pointing condition (312 ms $\pm 24 \mathrm{~ms}$ ). There was no main effect of vision condition ( $p=.13)$ and no interaction effect $(p=.08)$. 
Regarding the MT-data, the analysis revealed significant main effects of both task, $F(1,11)=21.02$, $p=.001, \eta_{p}^{2}=.66$, and vision condition, $F(1,11)=5.67, p=.038, \eta_{p}^{2}=.34$. Similar as in Experiment 1, movements were performed faster in the pro-pointing condition (407 ms $\pm 31 \mathrm{~ms}$ ) than in the antipointing condition (445 ms $\pm 35 \mathrm{~ms}$ ) and faster in the open-loop condition (403 ms $\pm 29 \mathrm{~ms}$ ) than after delay (449 ms $\pm 38 \mathrm{~ms})$. There was no interaction effect ( $p=.46)$.

\section{General Discussion}

The aim of the present study was to investigate if different movements, that according to the perception-action model recruit the perceptual mechanisms of the ventral stream (i.e. movements performed based on memory and movements based on spatial imagery), are affected similarly by visual illusions. As described in the introduction, the perception-action model would predict that goal-directed pro-pointing movements performed in an open-loop fashion (such that visual information is available during movement programming) should be unaffected by the visual illusion, while pro-pointing movements performed after delay as well as anti-pointing should be susceptible to illusion effects (Westwood \& Goodale, 2003). Furthermore, on the basis of the perception-action model, we would expect that the effects of introducing a delay and switching from pro-pointing to anti-pointing are non-additive as in all cases the movements are supposed to be mediated by the perceptual representations of the ventral stream. Our study yielded several interesting results that have both methodological as well as theoretical implications.

We will start our discussion by exploring the methodological implications of our findings. As depicted in Figures $2 \mathrm{~B}$ and $3 \mathrm{~A}$, uncorrected illusion effects seem to suggest that the effect of the illusion considerably (and significantly) decreases in the anti-pointing condition when a delay is introduced. This finding is not only unexpected but is in contrast to the predictions made by both the proponents and critics of the perception-action model. However, as confirmed by our results (see Figure 2A), the slope of the function relating shaft-length to pointing distance is steeper in the open-loop than in the delay conditions. This means increasing shaft-length by $10 \mathrm{~mm}$ increases the pointing amplitude 
by approximately $9 \mathrm{~mm}$ in the open-loop condition but only by $8 \mathrm{~mm}$ in the delay condition. Therefore, pointing movements in the delay condition are less responsive to physical changes in shaft-length. This finding can well explain why anti-pointing amplitudes are less affected by illusory length-changes in the delay conditions. In fact, when we correct for the slope differences, the unpredicted decrease in the illusion effect observed for delayed anti-pointing movement disappears. The issue that the size of the illusion effect depends on participants' responsiveness (slope) to physical variations in object size has been discussed in detail by Franz and colleagues (e.g., Franz, 2003a; Franz, Fahle, Bülthoff, \& Gegenfurtner, 2001; Franz et al., 2009; Franz et al., 2005). Franz and his colleagues argued that illusion effects must be corrected for differences in response functions before differences in the size of the illusion effect can be compared in a meaningful way. This recommendation for corrections has however been questioned by some researchers (e.g., Westwood \& Goodale, 2011). Specifically, Westwood and Goodale (2011) argued that the effect of illusions on target size estimations may be mediated by different mechanisms than the effect of physical size changes on target size estimations. In this case, it may not be appropriate to scale illusion effects based on the scaling factor obtained from measuring how size estimates related to true changes in target size as different scaling functions may underlie both tasks. In other words, Westwood and Goodale (2011) suggest that the response to physical object size changes and the response to illusory object size changes are two qualitatively different signals that cannot be easily related to each other. As pointed out by Schenk et al. (2011) this criticism is in conflict with some of the assumptions they make in their own studies in which they investigate whether two physically differently sized objects presented within a size contrast illusion (hence being perceived as similar in size) elicit the same grasping response or not (for detailed explanation see section 2.3. in Schenk et al., 2011) . However, despite this debate it is important to note that the correction procedure has also been suggested and successfully applied by researchers supporting the claims of the perceptionaction model (e.g., Dewar \& Carey, 2006; Glover \& Dixon, 2002). 
Importantly, the findings presented in this paper further strengthen the case for corrections. Specifically, we demonstrate that employing a scaling procedure can be essential in order to obtain consistent findings across different experimental conditions. This is especially nicely illustrated in our results of Experiment 2 in which a significant increase in the illusion effect after delay in pro-pointing (as predicted by the perception-action model) became only apparent after the effects were corrected for differences in the underlying response functions.

Remarkably, until now, the matter of using corrected illusion effects was exclusively discussed as an issue occurring when grasping performance is compared to performance in perceptual tasks in the context of perception-action dissociations. Here, we show for the first time that scaling illusion effects for differences in response functions is also essential for pointing movements. Furthermore, we show that these corrections can also be critical for within-category comparisons (i.e. when comparing performance across different movement conditions). Without a correction procedure we may end up not only with questionable perception-action dissociation but also with bogus perception-perception dissociations. Consequently, we suggest that whenever we want to find out how a behavioural measure (such as pointing, grasping or perceptual size estimation) relates to the participants' internal estimate of size in absolute values (e.g., $\mathrm{mm}$ ) we need to take into account the underlying response function. Generally speaking, whenever we aim to compare the same effects (e.g., size of illusion) between different modalities (e.g., perception vs. action) or between different tasks (e.g. pointing vs. anti-pointing), we have to find ways to make this comparison as valid and reliable as possible. In the existing literature, many theoretical conclusions (specifically about differences between the action and perception system) are based on such comparisons and make implicitly the assumption that all measures respond similarly to physical size variations. Here, we suggest that it is necessary to measure participants' response function to object size in all conditions and then correct the illusion effects accordingly to guarantee, or at least increase, the validity of these comparisons. In line with our own recommendation, we corrected the illusion effects in our study and base our interpretation solely on the corrected results. 
In the following, we discuss the implications of our findings for the perception-action model. In the pro-pointing conditions, we observed a small but significant illusion effect in the open-loop viewing condition. As expected, the illusion effect increased considerably when a 2 second delay was introduced. Even though the finding that illusion effects are significantly different from zero in the open-loop condition is in contrast with the predictions of a strong version of the perception-action model (no illusion effects when real-time programming is possible), the finding is in line with many other studies investigating grasping movements toward stimuli embedded in the Müller-Lyer figure (Franz et al., 2009; Heath, Rival, Westwood, \& Neely, 2005; Westwood et al., 2001). Like our study, these studies occluded vision at movement onset hence engaging the real-time mechanisms of the dorsal stream for movement programming. Two different accounts have been put forward to explain this inconsistency. In order to reconcile the finding with the perception-action model, it has been suggested that in situations in which participants are aware of the fact that visual information will be unavailable during movement execution (blocked design), they adopt the strategy of specifying all movement parameters offline and well before movement initiation is required (see, Heath et al., 2005). Hence, the spatiotemporal characteristics of the movement are no longer based on the realtime mechanisms of the dorsal stream but instead on the perception-based visual representation of the ventral stream that is susceptible to the illusion.

In contrast, Franz et al. (2009) argued that illusion effects occur in open-loop conditions as visual feedback about the hand relative to the target stimulus is missing. Hence, there is no opportunity for on-line corrections to occur during movement execution (in contrast to closed-loop movements). They conclude that actions are always guided by the same size representation and that there is no evidence for a shift from dorsal to ventral stream control depending on whether or not the target is visible during movement programming. According to Franz and colleagues (2009), the size of the illusion effect on actions increases when the amount of visual feedback available during movement execution decreases. 
Interestingly, both accounts would however predict (for different reasons) that illusion effects should be of similar size in the open-loop and the delay condition. Heath and colleagues (2005) assume that both tasks rely on the same illusion-distorted representation of the ventral stream and consequently the same illusion effects are to be expected in both conditions. In contrast, Franz and colleagues (2009) identify the availability of visual feedback as the critical variable. Since visual feedback is largely absent for both open-loop and delayed pointing similar illusion effects are expected. In contrast to both sets of predictions, our data shows that illusion effects are much larger in the delayed pro-pointing condition as compared to the open-loop pro-pointing condition

On the other hand, our findings are in line with studies that looked at the effect of delays on movement kinematics in experiments without visual illusions. These studies also found a specific effect of delays (that were different from those of open-loop movements) on movement accuracy (e.g., Bradshaw \& Watt, 2002; Heath \& Binsted, 2007; Hesse \& Franz, 2009; Westwood, Heath, \& Roy, 2003). The differences in movement kinematics between open-loop and delay conditions suggest that visual feedback alone is not the only factor that determines the size of the illusion effect in visuomotor actions. Potentially, illusion effects may additionally be moderated by visual memory of the target position and target size (see also, Hesse \& Franz, 2009; Hesse \& Franz, 2010). Moreover, our findings also suggest that there are task-related differences in illusion effects (between pro-pointing and anti-pointing) which cannot be attributed to the presence or absence of visual feedback. The perception-action model with its assumption of an illusion-susceptible ventral stream and an illusion-immune dorsal stream can account for the observed task-related differences in illusion effects but it cannot easily explain why the difference in illusion effects between two ventral tasks, namely delayed pro-pointing versus anti-pointing, is twice as large as the corresponding difference between a dorsal (open-loop pro-pointing) and a ventral task (delayed propointing). 
In our opinion, a more plausible explanation for the pattern of results is related to how the MüllerLyer illusion is supposed to work (Gillam \& Chambers, 1985; Mack et al., 1985; Smeets et al., 2002). According to this notion, pointing to the end-points of the Müller-Lyer figure can be based on two different sources of information: position and extent. While the illusion only induces a small misperception of position, its effect on the estimation of spatial extent is much larger (Gillam \& Chambers, 1985; Mack et al., 1985). In our experiment, the relevance of information about position and extent depended strongly on the required task. In the anti-pointing task, participants had to compute the target position for their pointing movement on the basis of the length of the shaft, hence they had to rely on the estimate of extent which is largely deceived by the illusion. As the estimation of extent is assumed to be affected by the fin configuration on either side of the shaft (Predebon, 2000; Redding \& Hawley, 1993), we hypothesized that illusion effects observed in antipointing should be halved when one of the fin configurations is removed (Experiment 2). This prediction was well supported by our data.

In contrast, in the pro-pointing conditions, participants could determine the target location based on spatial extent as well as based on position information which is less affected by the illusion. We therefore expect extent and position information would be integrated in a fashion that optimized the precision of the overall response therefore giving greater weight to more reliable and relevant cues. In fact, previous research has suggested that cues with high task-relevance and high reliability will be given greater weight than cues which are less relevant for the task, or are less reliable, when they are combined within one modality (e.g., for depth perception, see Landy, Maloney, Johnston, \& Young, 1995) as well as across different modalities (e.g., vision and touch, see Ernst \& Bülthoff, 2004). Our findings provide some tentative indication that a similar process may occur for the combination of positon and distance information. In the open-loop pro-pointing condition information about position will be relevant and reliable as participants could see the target position during movement programming. Therefore, the pro-pointing response should primarily rely on (relatively accurate) position information. Consistent with this explanation we found that the size of 
the illusion effect was very similar in conditions in which fins were present (Experiment 1) or absent (Experiment 2) at the start position.

Finally, in the delayed pro-pointing conditions, participants had to wait 2 seconds before they were allowed to start their movement. Since the delay will reduce the precision of the target position estimate (e.g., due to eye-movements occurring during the delay phase), participants are more likely to base their pointing movements on a weighted combination of both position and extent. Again, this is well supported by our findings which showed an intermediate illusion effect (larger than propointing open-loop but smaller than anti-pointing) in both experiments. In fact, the observed illusion effect can be almost perfectly described as a linear combination of about $65 \%$ position effect (as measured in open-loop pro-pointing) and 35\% extent effect (as measured in the anti-pointing task) in both Experiment 1 and 2. In Experiment 1 the estimate based on the data obtained in the openloop pro-pointing condition and the anti-pointing conditions would predict an illusion effect of 19.0 $\mathrm{mm}$ (19.5 mm measured) and in Experiment 2 of $10.6 \mathrm{~mm}$ (11 mm measured) for the pro-pointing delay condition. In summary, the hypothesis that the size of the observed illusion effects can be attributed to the fact that different visual cues are available for pro-pointing and anti-pointing and that the available visual cues obtain a different weighting after delay provides a good explanation for our findings. Interestingly, it was remarked by one reviewer that the perception-action model may well be able to accommodate our findings if the idea that humans combine different sources of information optimally (i.e. visual information about position and extent) would be incorporated into the model. Generally, we agree with the suggestion that by increasing the amount of assumptions (more degrees of freedom), the general idea of the model could be sustained (i.e. differential processing of visual information for perception and action). However, we think that for now the assumption that the same visual information is combined to serve both perception and action in an optimal manner provides a more parsimonious explanation for our findings than the perceptionaction model with some additional adjustments. 
In summary, our experiments show that it is important to correct for differences in response functions of the different performance measures (dependent variables) when we want to compare illusion effects across tasks. Franz and his colleagues already demonstrated that such differences can confound perception-action contrasts when grasping (action) and manual estimation (perception) are compared (Franz, 2003a; Franz \& Gegenfurtner, 2008). In this study, we went one step further and showed that differences in response functions are also relevant for pointing and do not only exist between perceptual and visuomotor tasks but also between different perceptual tasks. Secondly our findings are incompatible both with the hypothesis that task-dependent differences in illusion effects are experimental artefacts but also with the assumption that illusion effects are best explained in terms of the perception-action framework. Instead we argue that task-related differences in illusion effects are best explained within a framework that assumes that visual cues are combined across the visual system in a way that takes into account the task-relevance and reliability of a given cue (for a more detailed discussion, see Schenk, 2010). 


\section{References}

Aglioti, S., DeSouza, J. F. X., \& Goodale, M. A. (1995). Size-contrast illusions deceive the eye but not the hand. Current Biology, 5(6), 679-685.

Bradshaw, M. F., \& Watt, S. J. (2002). A dissociation of perception and action in normal human observers: the effect of temporal-delay. Neuropsychologia, 40, 1766-1778.

Brainard, D. H. (1997). The psychophysics toolbox. Spatial Vision, 10(4), 433-436.

Bruno, N., \& Bernardis, P. (2003). When does action resist visual illusions? Effector position modulates illusory influences on motor responses. Experimental Brain Research, 151, 225237.

Bruno, N., Bernardis, P., \& Gentilucci, M. (2008). Visually guided pointing, the Müller-Lyer illusion, and the functional interpretation of the dorsal-ventral split: conclusions from 33 independent studies. Neuroscience \& Biobehavioral Reviews, 32(3), 423-437.

Bruno, N., \& Franz, V. H. (2009). When is grasping affected by the Mueller-Lyer illusion? A quantitative review. Neuropsychologia, 47, 1421-1433.

Buonaccorsi, J. P. (2001). Fieller's theorem. In A. H. El-Shaarawi \& W. W. Piegorsch (Eds.), Encyclopedia of Environmetrics (pp. 773-775): Wiley, New York.

Dassonville, P., \& Bala, J. K. (2004). Perception, action, and Roelofs effect: A mere illusion of dissociation. PLoS Biology, 2(11), 1936-1945.

Dewar, M. T., \& Carey, D. P. (2006). Visuomotor 'immunity' to perceptual illusion: A mismatch of attentional demands cannot explain the perception-action dissociation. Neuropsychologia, 44, 1501-1508.

Ernst, M. O., \& Bülthoff, H. H. (2004). Merging the senses into a robust percept. Trends in Cognitive Sciences, 8, 162-169.

Fiehler, K., Bannert, M. M., Bischoff, M., Blecker, C., Stark, R., Vaitl, D., . . Rosler, F. (2011). Working memory maintenance of grasp-target information in the human posterior parietal cortex. Neuroimage, 54(3), 2401-2411. doi: 10.1016/j.neuroimage.2010.09.080

Fieller, E. C. (1940). The biological standardization of insulin. Supplement to the Journal of the Royal Statistical Society, 7(1), 1-64.

Franz, V. H. (2001). Action does not resist visual illusions. Trends in Cognitive Sciences, 5(11), 457459.

Franz, V. H. (2003a). Manual size estimation: a neuropsychological measure of perception? Experimental Brain Research, 151(4), 471-477.

Franz, V. H. (2003b). Planning versus online control: dynamic illusion effects in grasping? Spatial vision, 16(3-4), 211-224. 
Franz, V. H. (2004a). Is there a dynamic illusion effect in the motor system? Behavioral and Brain Sciences, 27(01), 34-35.

Franz, V. H. (2004b). The Optotrak Toolbox Retrieved April 15, 2010

Franz, V. H. (2007). Ratios: A short guide to confidence limits and proper use. (arXiv:0710.2024).

Franz, V. H., Fahle, M., Bülthoff, H. H., \& Gegenfurtner, K. R. (2001). Effects of visual illusions on grasping. Journal of Experimental Psychology: Human Perception and Performance, 27(5), 1124-1144.

Franz, V. H., \& Gegenfurtner, K. (2008). Grasping visual illusions: Consistent data and no dissociation. Cognitive Neuropsychology, 25(7-8), 920-950. doi: 10.1080/02643290701862449

Franz, V. H., Gegenfurtner, K. R., Bülthoff, H. H., \& Fahle, M. (2000). Grasping visual illusions: No evidence for a dissociation between perception and action. Psychological Science, 11(1), 2025.

Franz, V. H., Hesse, C., \& Kollath, S. (2009). Visual illusions, delayed grasping, and memory: No shift from dorsal to ventral control. Neuropsychologia, 47(6), 1518-1531. doi:

10.1016/j.neuropsychologia.2008.08.029

Franz, V. H., Scharnowski, F., \& Gegenfurtner, K. (2005). Illusion effects on grasping are temporally constant not dynamic. Journal of Experimental Psychology: Human Perception and Performance, 31(6), 1359-1378.

Gentilucci, M., Chieffi, S., Daprati, E., Saetti, M. C., \& Toni, I. (1996). Visual illusion and action. Neuropsychologia, 34(5), 369-376.

Gillam, B., \& Chambers, D. (1985). Size and position are incongruous: Measurements on the MüllerLyer figure. Perception \& Psychophysics, 37(6), 549-556.

Glover, S., \& Dixon, P. (2002). Dynamic effects of the Ebbinghaus Illusion in grasping: Support for a planning/control model of action. Perception \& Psychophysics, 64(2), 266-278.

Goodale, M. A., Kroliczak, G., \& Westwood, D. A. (2005). Dual routes to action: Contributions of the dorsal and ventral streams to adaptive behavior. Progress in Brain Research, 149, 269-283.

Goodale, M. A., \& Westwood, D. A. (2004). An evolving view of duplex vision: Separate but interacting cortical pathways for perception and action. Current Opinion in Neurobiology, 14, 203-211.

Goodale, M. A., Westwood, D. A., \& Milner, A. D. (2004). Two distinct modes of control for objectdirected action. Progress in Brain Research, 144, 131-144.

Haffenden, A. M., \& Goodale, M. A. (1998). The effect of pictorial illusion on prehension and perception. Journal of Cognitive Neuroscience, 10(1), 122-136. 
Haffenden, A. M., Schiff, K. C., \& Goodale, M. A. (2001). The dissociation between perception and action in the Ebbinghaus illusion: Nonillusory effects of pictorial cues on grasp. Current Biology, 11(3), 177-181.

Heath, M., \& Binsted, G. (2007). Visuomotor memory for target location in near and far reaching spaces. Journal of motor behavior, 39(3), 169-177.

Heath, M., Maraj, A., Gradkowski, A., \& Binsted, G. (2009). Anti-pointing is mediated by a perceptual bias of target location in left and right visual space. Experimental Brain Research, 192(2), 275-286.

Heath, M., Neely, K. A., Krigolson, O., \& Binsted, G. (2010). Memory-guided reaching: What the visuomotor system knows and how long it knows it. In D. Elliott \& M. A. Khan (Eds.), Vision and goal-directed movement: neurobehavioural perspectives (pp. 79-97). Champaign, IL: Human Kinetics.

Heath, M., Rival, C., Westwood, D. A., \& Neely, K. (2005). Time course analysis of closed-and openloop grasping of the Müller-Lyer illusion. Journal of Motor Behavior, 37(3), 179-185.

Hesse, C., \& Franz, V. H. (2009). Memory mechanisms in grasping. Neuropsychologia, 47(6), 15321545. doi: 10.1016/j.neuropsychologia.2008.08.012

Hesse, C., \& Franz, V. H. (2010). Grasping remembered objects: Exponential decay of the visual memory. Vision Research, 50(24), 2642-2650. doi: 10.1016/j.visres.2010.07.026

Hesse, C., \& Schenk, T. (2014). Delayed action does not always require the ventral stream: A study on a patient with visual form agnosia. Cortex, 54, 77-91.

Himmelbach, M., \& Karnath, H. O. (2005). Dorsal and ventral stream interaction: Contributions from optic ataxia. Journal of Cognitive Neuroscience, 17, 632-640.

Hu, Y., \& Goodale, M. A. (2000). Grasping after a delay shifts size-scaling from absolute to relative metrics. Journal of Cognitive Neuroscience, 12(5), 856-868.

International vocabulary of metrology - Basic general concepts and assocated terms (2012).

Kleiner, M. (2010). Visual stimulus timing precision in Psychtoolbox-3: Tests, pitfalls and solutions. Perception, 39, 189.

Landy, M. S., Maloney, L. T., Johnston, E. B., \& Young, M. (1995). Measurement and modeling of depth cue combination: In defense of weak fusion. Vision Research, 35(3), 389-412.

Mack, A., Heuer, F., Villardi, K., \& Chambers, D. (1985). The dissociation of position and extent in Müller-Lyer figures. Perception \& Psychophysics, 37, 335-344.

Maraj, A., \& Heath, M. (2010). Antipointing: perception-based visual information renders an offline mode of control. Experimental Brain Research, 202(1), 55-64. 
McIntosh, R. D., \& Schenk, T. (2009). Two visual streams for perception and action: Current trends. Neuropsychologia, 47(6), 1391-1396.

Milgram, P. (1987). A spectacle-mounted liquid-crystal tachistoscope. Behavior Research Methods, Instruments, \& Computers, 19(5), 449-456.

Miller, R. G. J. (1986). Beyond ANOVA: basics of applied statistics. New York: John Wiley.

Milner, A. D., \& Goodale, M. A. (1995). The visual brain in action: Oxford: Oxford University Press.

Milner, A. D., \& Goodale, M. A. (2006). The visual brain in action. (2nd ed.). Oxford: Oxford University Press.

Mon-Williams, M., \& Bull, R. (2000). The Judd illusion: Evidence for two visual streams or two experimental conditions? Experimental Brain Research, 130, 273-276.

Pavani, F., Boscagli, I., Benvenuti, F., Rabuffetti, M., \& Farnè, A. (1999). Are perception and action affected differently by the Titchener circles illusion? Experimental Brain Research, 127, 95101.

Post, R. B., \& Welch, R. B. (1996). Is there dissociation of perceptual and motor responses to figural illusions? Perception, 25(5), 569-581.

Prablanc, C., Echallier, J. F., Komilis, E., \& Jeannerod, M. (1979). Optimal response of eye and hand motor systems in pointing. Biological Cybernetics, 45, 113-124.

Predebon, J. (2000). Length illusions in conventional and single-wing Müller-Lyer stimuli. Perception \& Psychophysics, 62(5), 1086-1098.

Predebon, J. (2005). Manual-aiming bias and the Müller-Lyer illusion: The roles of position and extent information. Experimental Brain Research, 163, 100-108.

Redding, G. M., \& Hawley, E. (1993). Length illusion in fractional Müller-Lyer stimuli: An objectperception approach. Perception, 22, 819-828.

Schenk, T. (2010). Visuomotor robustness is based on integration not segregation. Vision Research, $50(24), 2627-2632$.

Schenk, T., Franz, V. H., \& Bruno, N. (2011). Vision-for-perception and vision-for-action: Which model is compatible with the available psychophysical and neuropsychological data? Vision Research, 51(8), 812-818. doi: 10.1016/j.visres.2011.02.003

Smeets, J. B. J., \& Brenner, E. (1999). A new view on grasping. Motor Control, 3, 237-271.

Smeets, J. B. J., \& Brenner, E. (2006). 10 years of illusions. Journal of Experimental Psychology: Human Perception and Performance, 32, 1501-1504.

Smeets, J. B. J., Brenner, E., de Grave, D. D. J., \& Cuijpers, R. H. (2002). Illusions in action: Consequences of inconsistent processing of spatial attributes. Experimental Brain Research, $147,135-144$. 
Soechting, J. F., \& Flanders, M. (1989). Sensorimotor representations for pointing to targets in threedimensional space. Journal of Neurophysiology, 62(2), 582-594.

Welch, R. B., Post, R. B., Lum, W., \& Prinzmetal, W. (2004). The relationship between perceived length and egocentric location in Müller-Lyer figures with one versus two chevrons. Perception \& Psychophysics, 66(7), 1095-1104.

Westwood, D. A., \& Goodale, M. A. (2003). Perceptual illusion and the real-time control of action. Spatial Vision, 16(3-4), 243-254.

Westwood, D. A., \& Goodale, M. A. (2011). Converging evidence for diverging pathways: Neuropsychology and psychophysics tell the same story. Vision Research, 51(8), 804-811.

Westwood, D. A., Heath, M., \& Roy, E. A. (2000). The effect of a pictorial illusion on closed-loop and open-loop prehension. Experimental Brain Research, 134(4), 456-463.

Westwood, D. A., Heath, M., \& Roy, E. A. (2003). No evidence for accurate visuomotor memory: systematic and variable error in memory-guided reaching. Journal of Motor Behavior, 35(2), 127-133.

Westwood, D. A., McEachern, T., \& Roy, E. A. (2001). Delayed grasping of a Mueller-Lyer figure. Experimental Brain Research, 141, 166-173. 
Table 1:

Mean illusion effects in $\mathrm{mm}$ for both shaft lengths (SEM) in Experiment 1 and 2.

\begin{tabular}{lrrrr}
\hline Shaft length & \multicolumn{2}{c}{$70 \mathrm{~mm}$} & \multicolumn{2}{c}{$100 \mathrm{~mm}$} \\
& \multicolumn{1}{c}{ Exp. 1 } & \multicolumn{1}{c}{ Exp. 2 } & \multicolumn{1}{c}{ Exp. 1 } & \multicolumn{1}{c}{ Exp. 2 } \\
\hline Pro-Pointing - OL & $8.4(0.99)$ & $6.8(0.97)$ & $9.7(1.12)$ & $7.8(0.77)$ \\
Pro-Pointing - DL & $14.5(1.59)$ & $7.7(1.56)$ & $17.3(2.21)$ & $9.5(1.39)$ \\
Anti-Pointing - OL & $30.4(1.11)$ & $13.9(1.40)$ & $40.0(2.24)$ & $17.0(0.76)$ \\
Anti-Pointing - DL & $24.4(1.64)$ & $11.4(1.15)$ & $32.2(2.93)$ & $13.6(1.37)$ \\
\hline
\end{tabular}




\section{Figure legends}

Figure 1: Illustration of the stimuli and task of the participants. Pointing movements always started from the middle of the screen. The Müller-Lyer illusion was either displayed upward or downward relative to the start position of the hand. Figure $1 \mathrm{~A}$ provides depicts the procedure in the propointing task and Figure $1 \mathrm{~B}$ the procedure in the anti-pointing task. For more information see methods section.

Figure 2: Experiment 1: Average pointing distance and illusion effects. Figure 2A depicts the average pointing distance as a function of the illusory context (fins-in vs. fins-out), vision condition (openloop vs. delay) and the actual shaft length $(70 \mathrm{~mm}$ and $100 \mathrm{~mm}$ ). Figure $2 \mathrm{~B}$ depicts the average (uncorrected) illusion effects across all participants. Figure $2 \mathrm{C}$ depicts the corrected illusion effects that take into account the participants' response function to physical changes in shaft length. Error bars depict \pm 1 SEM (for further details see methods section).

Figure 3: Experiment 2: illusion effects. Figure $3 \mathrm{~A}$ shows the average uncorrected illusion effects (calculated as the mean difference between fin-out and fin-in conditions) across both tasks (propointing and anti-pointing) and vision conditions (open-loop vs. $2 \mathrm{~s}$ delay). Figure $3 \mathrm{~B}$ shows the corrected illusion effects. Note that the range of the $y$-axis is halved as compared to Figure $2 \mathrm{~B}$ and 2C. Error bars depict \pm 1 SEM. 

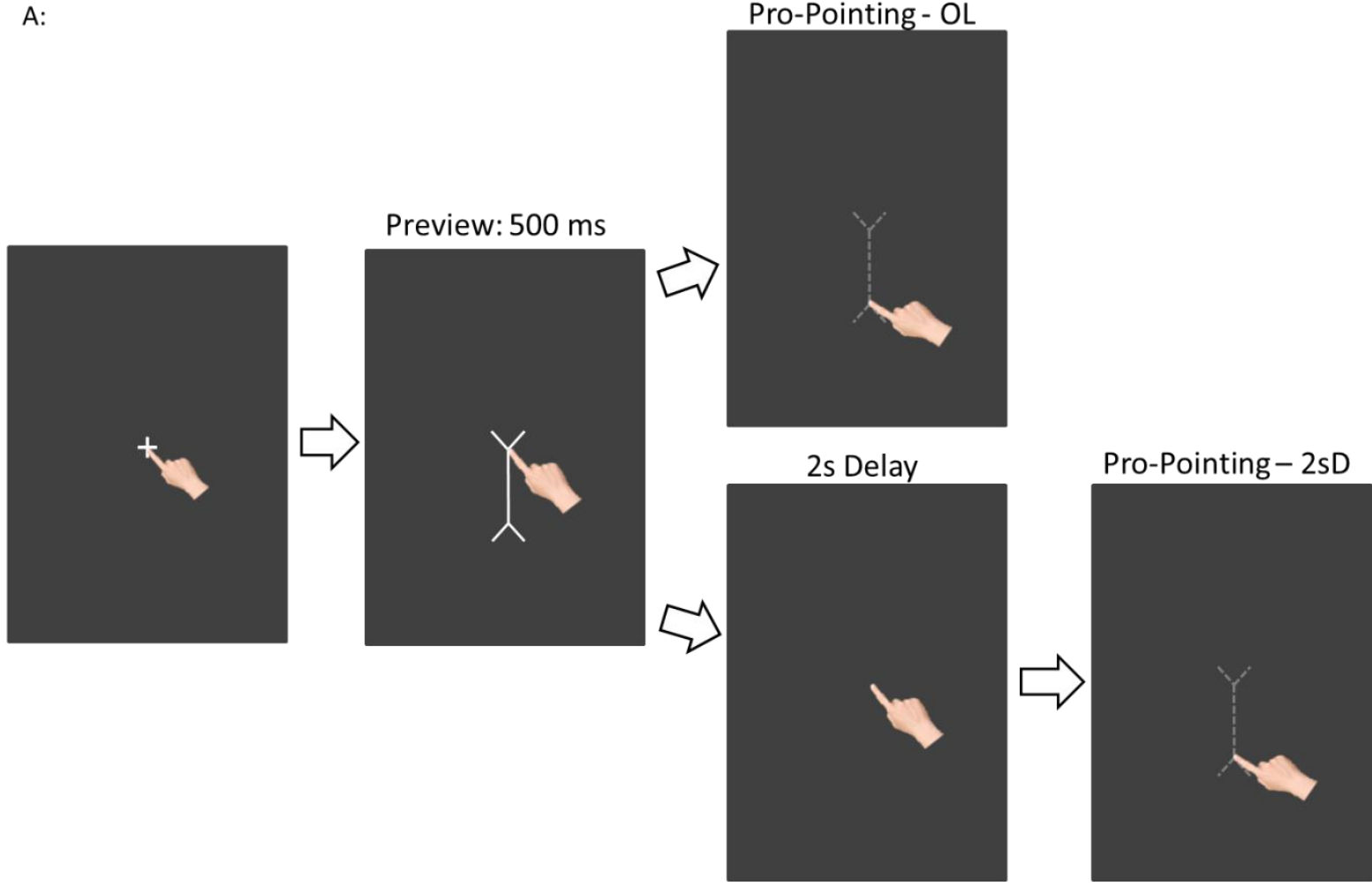

B:

Anti-Pointing - OL
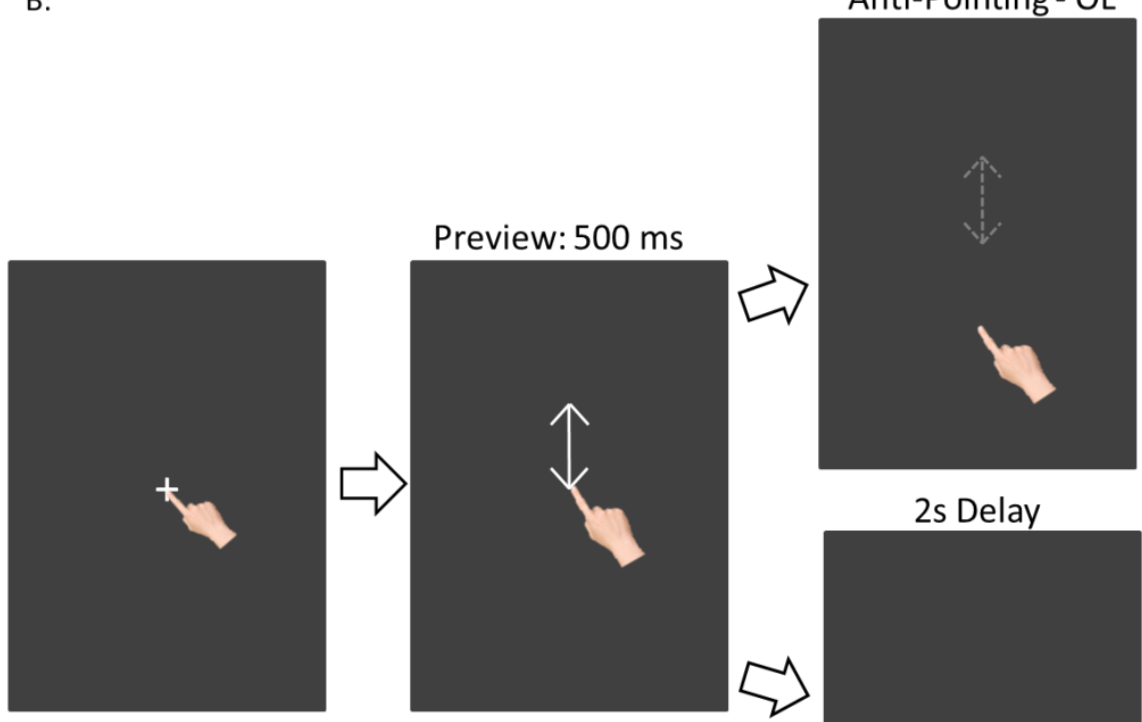

2s Delay

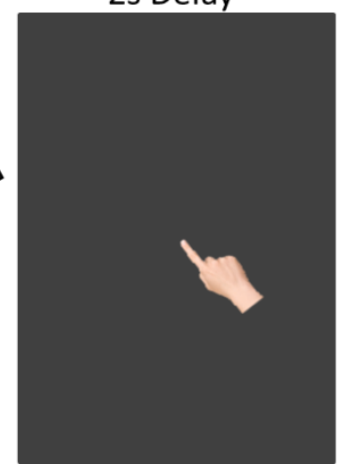

Anti-Pointing - 2sD

Figure 1 

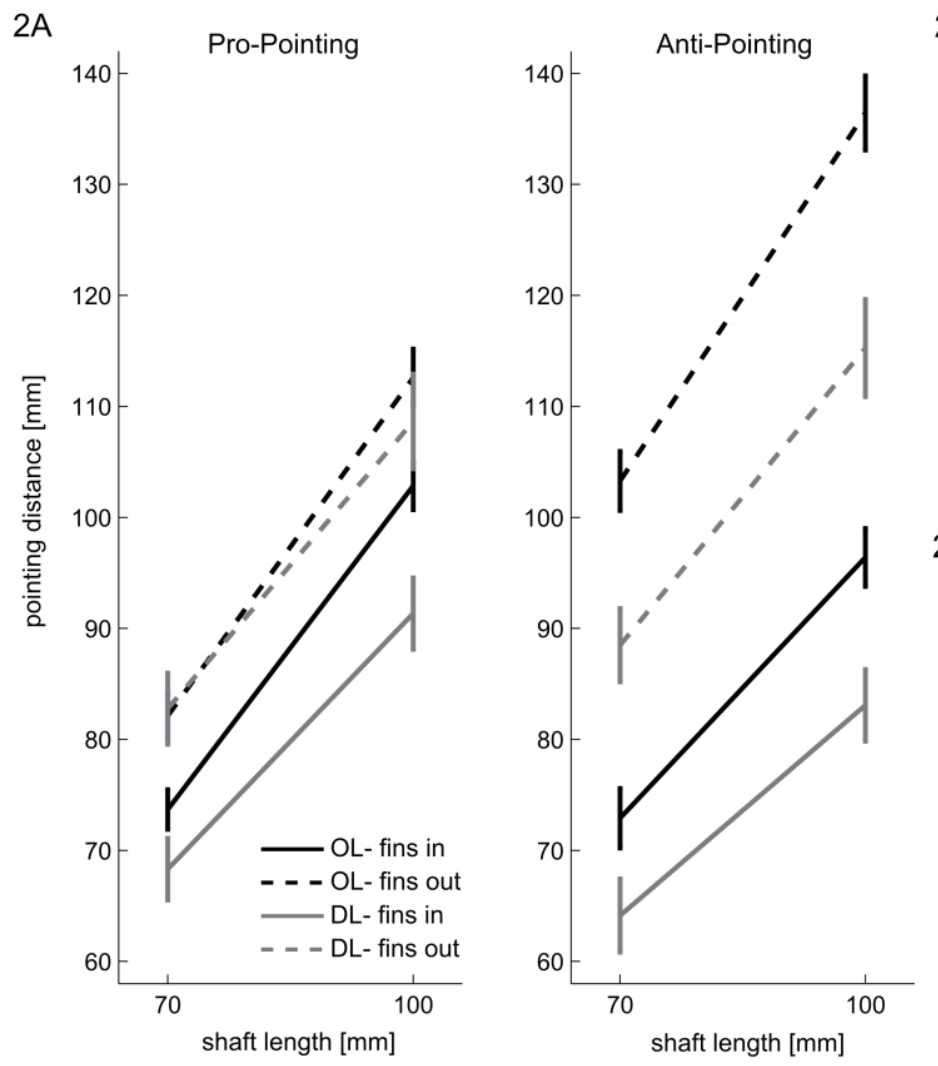

2B

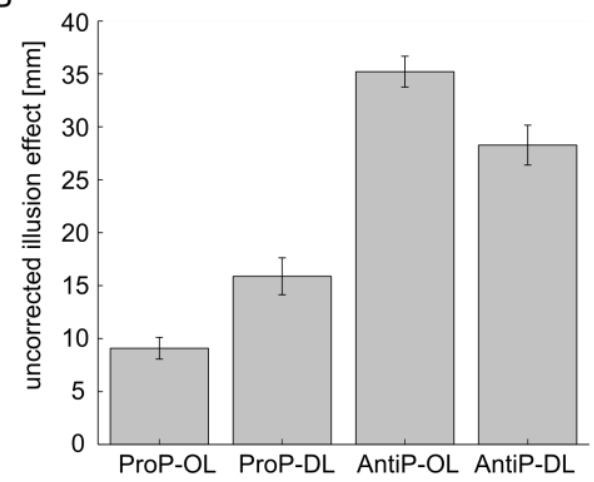

$2 \mathrm{C}$

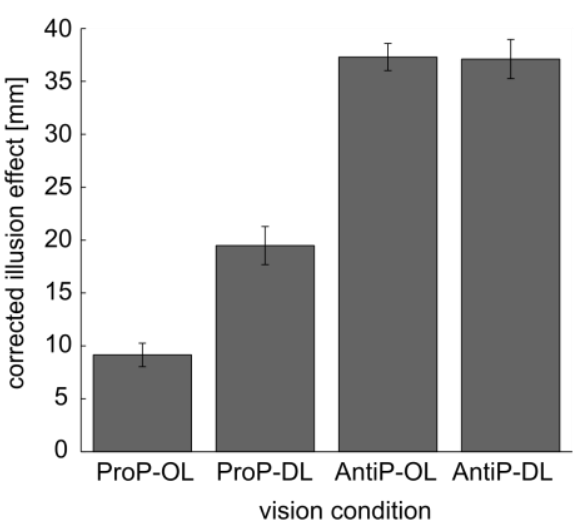

Figure 2 

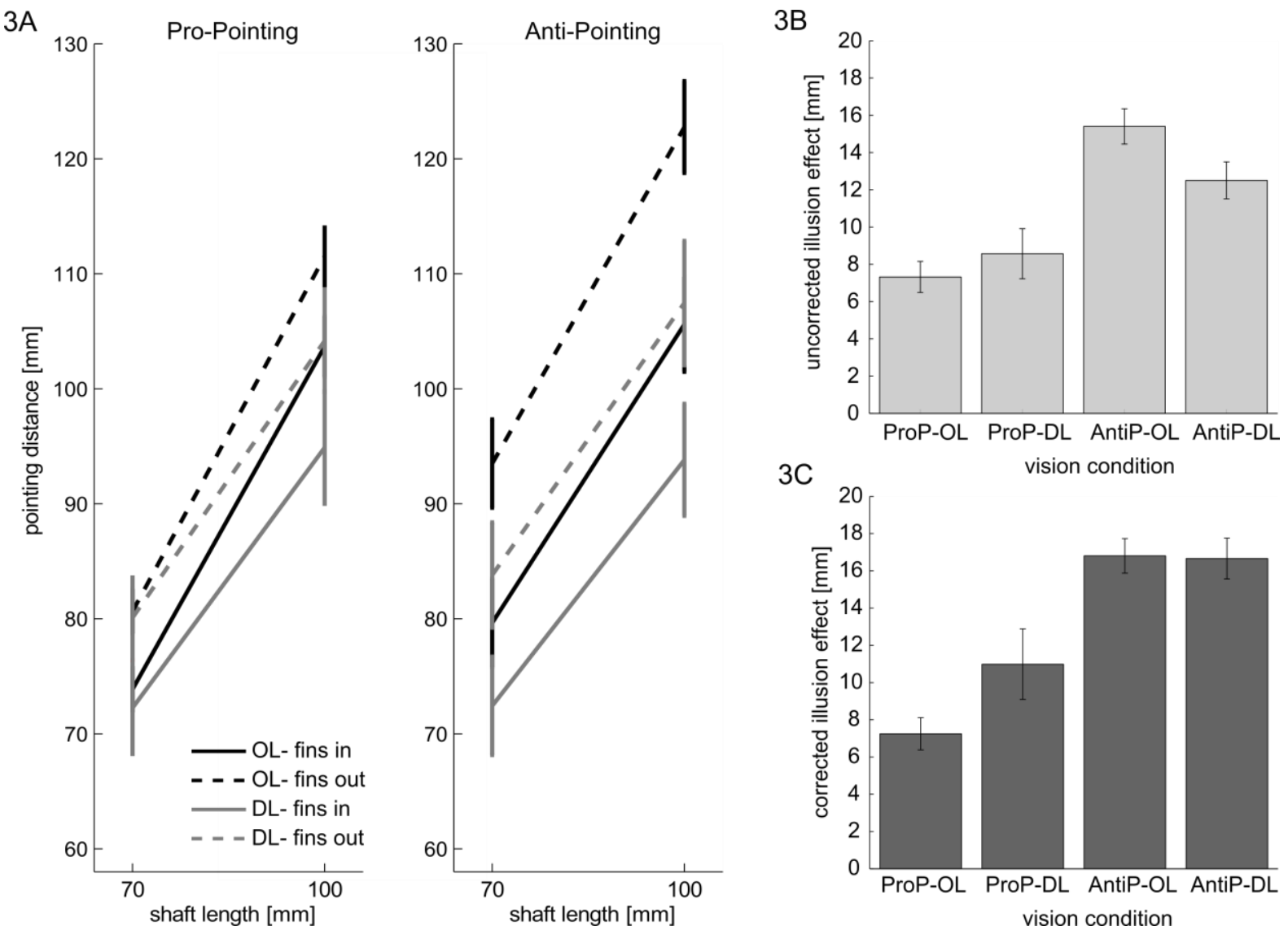

Figure 3 